306 UNIQUE ASSOCIATION OF HLA-DRB1*15, HLA DRB1*03 AND EBV WITH ADULT AND PAEDIATRIC SYSTEMIC LUPUS ERYTHEMATOSUS (SLE) PATIENTS IN NORTH INDIA

${ }^{1} \mathrm{P}$ Prabir Das, ${ }^{1} \mathrm{RW}$ Minz*, ${ }^{1} \mathrm{~S}$ Anand, ${ }^{2} \mathrm{~A}$ Sharma, ${ }^{1} \mathrm{~B}$ Saikia, ${ }^{3} \mathrm{~S}$ Singh. ${ }^{1}$ Postgraduate Institute of Medical Education and Research, Immunopathology, Chandigarh, India; ${ }^{2}$ Postgraduate Institute of Medical Education and Research, Internal Medicine, Chandigarh, India; ${ }^{3}$ Postgraduate Institute of Medical Education and Research, Advanced Paediatric Centre, Chandigarh, India

\subsection{6/lupus-2017-000215.306}

Background and aims SLE is multisystem autoimmune disease, having known HLA-DRB1*15 and DRB1*03 risk alleles and an association with EBV in the Caucasoid population. We compared the association of HLA-DRB1 and EBV in North Indian cohort of adult and paediatric SLE

Methods We analysed 109 adult SLE (aSLE) and 52 paediatric SLE (pSLE) with 278 age and sex matched control adult (CA) and paediatric (CP) for HLA-DRB1 genotyping by PCR-SSP, EBV-IgM and IgG to VCAgp125, VCAp19, EBNA-1, p22 and EA-D by line blot assay and EBV load by real-time PCR

Results The frequencies of DRB1*15 and DRB1*03 were higher in $\operatorname{SLE}(\mathrm{OR}=2.57$ and 1.67 respectively) compared to controls. aSLE had higher frequencies of DRB1*03 $(\mathrm{OR}=2.33)$ and $\mathrm{DRB} 1 * 04 \quad(\mathrm{OR}=7.51)$ compared to $\mathrm{pSLE}$ whereas, pSLE had higher frequency of DRB1*15 (OR=2.42) compared to aSLE. pSLE had more $3+$ to $4+$ positivity of EBV-IgG to VCAgp125 compared to aSLE $(p=0.0008)$. EBVIgM to VCAp19, EBNA-1 and EA-D $(25 \%, 12.5 \%$ and $6.25 \%$ respectively) were present only in pSLE. pSLE had higher EBV load compared to $\operatorname{aSLE}(\mathrm{p}=0.045)$. IgG to $\mathrm{p} 22$ and EA-D were associated with DRB1*03 in aSLE (OR $=3.92$ and 5.28 respectively) and IgG to VCAgp125, VCAp19, EBNA-1, p22 and EA-D were associated with DRB1*15 in pSLE $(\mathrm{OR}=4.0,3.44,4.6,4.8$ and 4.8 respectively)

Conclusions DRB1*15 and DRB1*03 are risk alleles in North Indian SLE. Both show strong associations with immune response to EBV proteins. pSLE has stronger association with DRB1*15, which is associated with early infection, stronger immune response to EBV proteins and higher EB viral load, which may explain more severity of pSLE

\section{THE PATHOGENIC MECHANISMS OF SYSTEMIC LUPUS ERYTHEMATOSUS ASSOCIATED GENES PNP, PLEKHF2 AND ANKRD44}

J Qian, S Nan*. RenJi Hospital- School of Medicine- Shanghai JiaoTong University, Shanghai Institute of Rheumatology, Shanghai, China

\subsection{6/lupus-2017-000215.307}

Background and aims To detect the expression of three SLEsusceptible genes, PNP, PLEKHF2 and ANKRD44 in SLE PBMC and lupus nephritis kidney samples, and to investigate their function.

Methods We collected PBMC from 46 SLE patients and 48 healthy controls, and renal biopsy tissues from 12 lupus nephritis patients and peri carcinomatous tissues from 10 patients with kidney cancer. The mRNA expression levels of PNP, PLEKHF2 and ANKRD44 were detected by qPCR. Their expression levels with the SLE clinical features and IFN scores were analysed. ANKRD44 was tested at different time points in Raw264.7 cells during IFN stimulation. The expression of
ANKRD44 was knockdown by using siRNA in Raw264.7 cells. The change of IFIGs and the activation of IFN signalling pathway were detected by Real-time PCR and western blotting.

Results PNP, PLEKHF2 and ANKRD44 were found significantly decreased in SLE PBMCs compared with healthy controls. The mRNA expression of PNP and ANKRD44 were significantly decreased while the expression of PLEKHF2 was increased in kidney of lupus nephritis. The expression of PNP was negatively correlated with IFN score in SLE PBMC samples, while the expression of ANKRD44 was negatively correlated with IFN score in lupus nephritis kidney. ANKRD44, which could be down-regulated by IFN alpha, could inhibit the type I IFN signalling pathway and downregulate the expression of IFIGs.

Conclusions PNP and ANKRD44, expressed abnormally and associated with IFN alpha, might be used as new candidate biomarkers for SLE diagnosis; ANKRD44 could repress the IFN downstream pathway, it would be a potential drug target.

\section{STUDY ON THE ROLE OF MICRORNA125A IN LUPUS DISEASE IN MICE}

${ }^{1} \mathrm{H}$ Qin*. ${ }^{1}$ Ren Ji Hospital- School of Medicine- Shanghai Jiao Tong University, Rheumatology, Shanghai, China

\subsection{6/lupus-2017-000215.308}

Background and aims To investigate whether over-expression of miRNA125a has an effect on organic injuries and its potential mechanisms by using miRNA-125a-agomir transfected MRL/lpr mice

Methods 5-week-old female MRL/lpr and MRL/n mice were divided into three groups : the lpr-miRNA-group, given miRNA-125a-agomir intravenously; the lpr-PBS-group, given PBS intravenously ; MRL-control-group: receiving no treatment. Blood samples and urine were collected weekly interval from 5-week-age. At 13-week-age and 17-week-age, bronchoalveolar lavage fluid, blood sample, lung and spleen tissues were collected and analysed in half of the mice.

Results MiRNA-125a levels in splenocytes were significantly elevated in MRL/lpr mice.

A variety of inflammatory cell infiltration, mostly $\mathrm{T}$ cells, in lung tissues was statistically alleviated in lpr-miRNA group.

Flow cytometry analysis indicated that in lpr-miRNA group, the proportion of splenic plasma cells in lpr-miRNA group was significantly decreased than that in lpr-PBS group.

Cytokines analysis showed serum levels of RANTES in lprmiRNA group were statistically reduced.

The serum level of anti-dsDNA and the high tilter proportions of ANA were much lower in lpr-miRNA group than in lpr-PBS group.

Conclusions Intravenous injection of miRNA125a-agomir +Engreeen in vivo transfection reagent mixture solution could transfect miRNA-125a and increase the level of miRNA-125a expression safely and effectively.

Over-expression of miRNA-125a could alleviate inflammatory cell infiltration in lung tissues in lpr mice and reduce the proportion of splenic plasma cell.

Elevation of miRNA-125a expression could inhibit the expression of RANTES in lpr mice, which in turn reduce the autoimmune inflammation to a certain extent. 
Over-expression of miRNA-125a could reduce serum autoantibodies and suppress autoimmune reaction in lpr mice.

\section{CORRELATION OF URINARY BIOMARKERS MCP1 AND NGAL WITH LUPUS NEPHRITIS (LN) HISTOLOGY AND DISEASE ACTIVITY SCORE}

R Ramamoorthy*, S Rajeswari, T Tamilselvam, M Kumudha. Madras Medical College, rheumatology, Chennai, India

\subsection{6/lupus-2017-000215.309}

Background and aims Treatment of $\mathrm{LN}$ requires an accurate assessment of disease activity.

This study aims to assess the correlation of urinary biomarkers MCP1 and NGAL with the disease activity in LN.

Methods Materials and Methods: Prospective study conducted in a tertiary care centre. 60 patients with SLE were recruited and divided into 3 groups: one with Active LN $(n=22)$, another with Inactive $\mathrm{LN}(\mathrm{n}=20)$ and third formed of SLE patients with no renal involvement $(n=18)$. Active $\mathrm{LN}$ patients underwent renal biopsy. For comparison another group of age and sex matched controls was taken $(n=20)$. Disease activity was correlated with biopsy and baseline characteristics. Urinary MCP1 and NGAL were measured and its correlation with disease activity was analysed.

Results In patients with active LN, both UMCP1/Cr and UNGAL/Cr were significantly elevated (92.78, $76.11 \mathrm{pg} / \mathrm{ml}$, $\mathrm{p}<0.001)$. In both control group and SLE without renal involvement the values of UMCP1/Cr and UNGAL/Cr were normal $(24.44,22.22 \mathrm{pg} / \mathrm{ml}$ in control and 24.3, $22.80 \mathrm{pg} / \mathrm{ml}$ in SLE without renal involvement). In patients with inactive $\mathrm{LN}$ the values of $\mathrm{UMCP} 1 / \mathrm{Cr}$ and UNGAL/Cr were observed to be significantly higher than control $(44.18,38.45 \mathrm{pg} / \mathrm{ml}$, $\mathrm{p}<0.005)$ and lower than those of active LN. Values of $\mathrm{UMCP} 1 / \mathrm{Cr}$ and UNGAL/Cr were found to be in close correlation with mean rSLEDAI scores of active LN (10) and inactive $\mathrm{LN}(3.6)$ and disease activity as per histopathology.

Conclusions Levels of urinary biomarkers UMCP1 and UNGAL were significantly elevated in active LN and found to have excellent correlation with histopathological disease activity index and rSLEDAI scores.

\section{STUDY ON THE ROLE AND MECHANISMS OF ABERRANT EXPRESSION OF TRANSCRIPTION FACTOR WT1 IN THE PSORIASIS VULGARIS LESION FORMATION}

W Ruifang*, Z Ming, S Yuwen, L Qianjin. The Second Xiangya Hospital of Central South University, Dermatology, Chang Sha, China

\subsection{6/lupus-2017-000215.310}

Background and aims Psoriasis vulgaris (PV) is a chronic inflammatory skin disease characterised by abnormal keratinocytes proliferation and apoptosis. Evidence has showed that transcription factor WT1 plays important role in many pathophysiologic processes such as organs development, tumorigenesis and cells proliferation. However, the role of WT1 in PV is still remain unclear. In this study, we will investigate the role of WT1 in the pathogenesis of lesion formation in PV.

Methods Skin specimens and peripheral blood mononuclear cells (PBMCs) were obtained from 25 patients with PV and
20 age- and sex-matched healthy subjects. mRNA and protein levels were detected by real-time RT-PCR and western blot. WT1 siRNA and WT1 overexpression plasmid were transfected into HaCaT cells with lipofectamine 2000 respectively. The proliferation and apoptosis of $\mathrm{HaCaT}$ cells were detected by CCK8 kit and Annexin V-FITC/PI Apoptosis Detection Kit

Results Compared with normal controls, both the mRNA and protein level of WT1 were increased significantly in psoriatic skin and PBMCs. Transfect with WT1 siRNA inhibited the proliferation of $\mathrm{HaCaT}$ cells and promoted $\mathrm{HaCaT}$ cells apoptosis, while WT1 overexpression plasmid exhibited the opposite effects on HaCaT cells. The global DNA methylation level of psoriatic skins and PBMCs were elevated accompanied with increased DNMT1 expression. In addition, an positive correlation was observed between WT1 and DNMT1.

Conclusions Increased WT1 promotes the keratinocytes proliferation and inhibites the apoptosis of keratinocytes which may mediated by recruiting DNMT1 to its target genes related to cell proliferation and apoptotic pathway.

\section{FAMILIALITY AND CONCORDANCE OF PRESENTING MANIFESTATIONS AMONG FILIPINO PATIENTS FROM LUPUS MULTIPLEX FAMILIES}

V Santos*, ST Navarra. University of Santo Tomas Hospital, Internal Medicine - Section of Rheumatology, Manila, Philippines

\subsection{6/lupus-2017-000215.311}

Background and aims To describe the familiality and concordance of manifestations among Filipino patients with systemic lupus erythematosus (SLE) and their affected first degree relatives (FDR).

Methods Filipino mulitplex SLE families with at least 2 first degree relatives (FDRs) diagnosed as SLE were identified from University of Santo Tomas (UST) Lupus Database. Demographic and disease characteristics were described, and types of relationships within families were analysed for concordance of presenting manifestations using McNemar and Fisher's Exact tests, with significance at $\mathrm{p}<0.05$.

Results The prevalence of familial SLE in the UST Lupus Database $(n=2474)$ was $7.8 \%$. There were 192 patients (173, 90\% females) from 95 families (2 families with 3 FDRs), including 25 parent-offspring pairs (23 mother-daughter) and 70 sibling pairs (56 sister-sister). Average age at SLE diagnosis was 31+11.4 SD (range 5-68) years for all affected FDRs. Among parent-offspring pairs, parents' age averaged $44.8+9.7$ SD (range 29-68) years and their offsprings averaged 23.6 +10.6 SD (range 5-35) years at SLE diagnosis, $\mathrm{p}<0.001$. Average age at SLE diagnosis among sibling pairs was 28.6 +11.4 SD (range 9-55) years, with a positive linear association of age at SLE diagnosis between siblings, $p<0.001$. Most common presenting manifestations were malar rash (47\%), oral ulcers (45\%), photosensitivity (40\%), hematologic (39.9\%) and arthritis (39\%). Concordance among related FDRs was significant for oral ulcers and hematologic manifestations, $\mathrm{p}<0.05$.

Conclusions This study underscores the role of genetics in age onset and clinical expression of lupus. 